\title{
Paroxysmal nocturnal hemoglobinuria (PNH): An atypical case report
}

\author{
Luc Longrée ${ }^{1}$, Renaud Roufosse ${ }^{1}$, Stéphanie Maréchal ${ }^{1}$, Julie Goffinet ${ }^{2}$, Renaud Maquet ${ }^{3}$, Christian Focan*1 $^{* 1}$ \\ ${ }^{1}$ Department of Onco-Hematology, CHC-Liège; Clinique Saint-Joseph, Liège, Belgium \\ ${ }^{2}$ Clinical Biology Laboratory, CHC-Liège; Clinique Saint-Joseph, Liège, Belgium \\ ${ }^{3}$ Department of Pharmacy, CHC-Liège; Clinique Saint-Joseph, Liège, Belgium
}

Received: December 23, 2017

DOI: $10.5430 /$ crim.v5n1p39
Accepted: January 29, $2018 \quad$ Online Published: February 9, 2018

URL: https://doi.org/10.5430/crim.v5n1p39

\begin{abstract}
Introduction: Paroxysmal nocturnal hemoglobinuria $(\mathrm{PNH})$ also called Marchiafava-Micheli syndrome is a rare disease (1.3 case per million inviduals). It is a clonal disorder caused by a medullary stem cells acquired mutations on the $P I G-A$ gene, inducing a partial or general deficit of proteins linking to cell membrane through a glycophosphatidylinositol (GPI) anchor.

Case presentation: We confirmed the diagnosis of PNH in a 35-year-old woman presenting a relatively well tolerated but progressive pancytopenia and also a concomitant cyanocobalamin deficiency. The diagnosis was classically obtained through flow cytometry determination of specific disease markers on red blood cells (CD55; CD59; FLAER). After a CD34 selected grafting from his fully HLA-compatible brother as donor, the patient entered in a sustained complete remission of PNH syndrome.

Conclusion: After bone-marrow grafting, prolonged complete remission (cure) of PNH presenting primarily as a medullary insufficiency, may be obtained.
\end{abstract}

Key Words: Paroxysmal nocturnal hemoglobinuria, Medullary aplasia, Allograft

\section{INTRODUCTION}

Paroxysmal nocturnal hemoglobinuria $(\mathrm{PNH})$ characterized by "dark nocturnal urines" has been first observed by the end of XIXth century and further better described by Marchiafava and Micheli in 1931. ${ }^{[1]}$ This is a rare disease (1.3 cases per million individuals ) expressed predominantly in young (median age 32) caucasian people. Recent studies have shown that the disease was related to an acquired mutation of hematopoietic stem cell on phosphatidyl-inositol glycan (GPI) class A (PIG-A) gene located on X chromosome. ${ }^{[2-6]}$ This mutation will lead to a partial or complete failure of proteins linked to cell membrane through a glycophosphatidylinositol $(G P I)$ anchor. $^{[2-6]}$ We report the case of a young woman whose initial presentation was atypical. The diagnosis was difficult because of concomitant cyanocobalamin ( B12 vitamin ) deficiency. She entered in a sustained complete remission of disease after allogenic bone marrow grafting.

\section{CASE PRESEntation}

Mrs J.V born on April 3rd 1978 was seen in the out patient clinic on April 29th 2013. Her previous history was characterized by psycho-functional problems while in 2001, she underwent a laparoscopic gastroplasty for morbid obesity. She complained from fatigue, dyspnea and occasional palpitations. There was no familial history of hematologi-

\footnotetext{
* Correspondence: Christian Focan; Email: christian.focan@chc.be; Address: Oncology Department, Clinique Saint-Joseph; rue de Hesbaye, 75; B-4000-Liège, Belgium.
} 
cal disorders. Medications were: amitriptyline, alprazolam, tetrazepam and prothipendyl. Clinical examination and general biology were normal but hematological parameters revealed pancytopenia and macrocytosis initially attributed to a effective cyanocobalamin (B12 vitamin) deficiency (see Table 1). Serum iron, ferritin, $\mathrm{LDH}$ and reticulocytes titrations remained within normal range as well as Coombs erythrocytes test with absence of circulating schizocytes. However haptoglobin level was broken down from onset. Despite a correction of B12 levels from repeated parenteral administrations, hematological parameters did not improve while patient mentioned the occurrence of dark nocturnal urines. Thus a bone marrow examination was performed on May 15th 2013. Megaloblastic cells were seen on red lineage with enlargement and irregularities of cytoplasm, nuclear abnormalities in pearl chromatin and Jolly bodies. White lineage was diminished with a right deviation and some nuclear alterations. Lympho-plasmocytosis was also increased due in a context of relative medullary hypoplasia (see Figure 1). The medullary hypoplasia with right deviation of red and granulocytic lineages and reactional lympho-plasmocytosis was confirmed by a second analysis on June 19th 2013 (see Figure 2). Prussian Blue coloration did not show ringed sideroblasts and there was no evidence for a myelodysplastic syndrome. Karyotyping was normal. The diagnosis of PNH was confirmed by flow cytometry determinations of specific PNH markers CD55, CD59 and aerolysin. ${ }^{[2-6]} \mathrm{A}$ small GPI-deficient clone of $7 \%$ in circulating blood and $19 \%$ on medullary cells was notified.

Table 1. Hematological parameters

\begin{tabular}{|c|c|c|c|c|c|c|c|}
\hline \multirow{2}{*}{ Variables; Units } & \multirow{2}{*}{ Normal values } & \multicolumn{2}{|c|}{ Pre Allograft } & \multirow{2}{*}{$\begin{array}{c}\text { Graft } \\
2013 / 11 / 14\end{array}$} & \multicolumn{3}{|c|}{ Post Allograft } \\
\hline & & $2013 / 4 / 8$ & 2013/9/11 & & $2014 / 5 / 5$ & 2014/12/2 & $2015 / 9 / 29$ \\
\hline $\mathrm{Hb} ; \mathrm{g} \%$ & $12.0-16.0$ & 7.6 & 8 & 9 & 11.1 & 11.6 & 12.2 \\
\hline Hematocrite; \% & $37-47$ & 23 & 24 & 28 & 31.8 & 36 & 37.2 \\
\hline $\begin{array}{l}\text { Erythrocytes; } \\
\text { million/mm } \mathrm{mm}^{3}\end{array}$ & 4.1-5.4 & 1.97 & 2.33 & 2.64 & 3.42 & 3.7 & 4.05 \\
\hline MCV; microns cube & $80-100$ & 116.7 & 103 & 106 & 93 & 97.3 & 91.9 \\
\hline MCH; pgr & $26-32$ & 38.5 & 34 & 34 & 32.5 & 31.4 & 30.1 \\
\hline Leucocytes & $4-9 * 1,000$ & 2 & 2.5 & 2.6 & 5.94 & 4.05 & 4.82 \\
\hline Neutrophiles \% & $50-70$ & 54 & 32.6 & 68.2 & 74.2 & 53.8 & 55.2 \\
\hline Platelest; * 1,000/mm ${ }^{3}$ & $150-400$ & 72 & 66 & 86 & 385 & 321 & 295 \\
\hline Serum iron; microgr/dl & $37-145$ & 129 & 157 & & 48 & 302 & 100 \\
\hline Ferritin; microgr/L & $10-291$ & 156 & 175 & & & & \\
\hline Vit B12; ng/L & 211-911 & 121 & 239 & & & & \\
\hline Haptoglobin; gr/L & $0.56-3.2$ & 0.02 & & & & & \\
\hline LDH; U/L & $<480$ & 386 & 380 & 361 & 173 & 209 & 153 \\
\hline
\end{tabular}

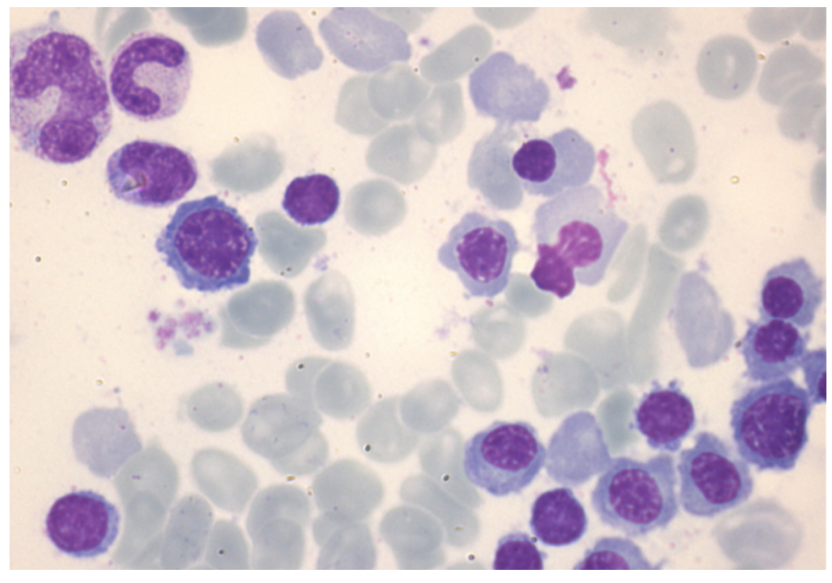

Figure 1. Medullary sample (15-5-2013); poor bone marrow; note a giant metamyelocyte and megaloblastic normoblasts with nuclear abnormalities (Hematoxylin-Eosin colouring; magnification $500 \times$ )

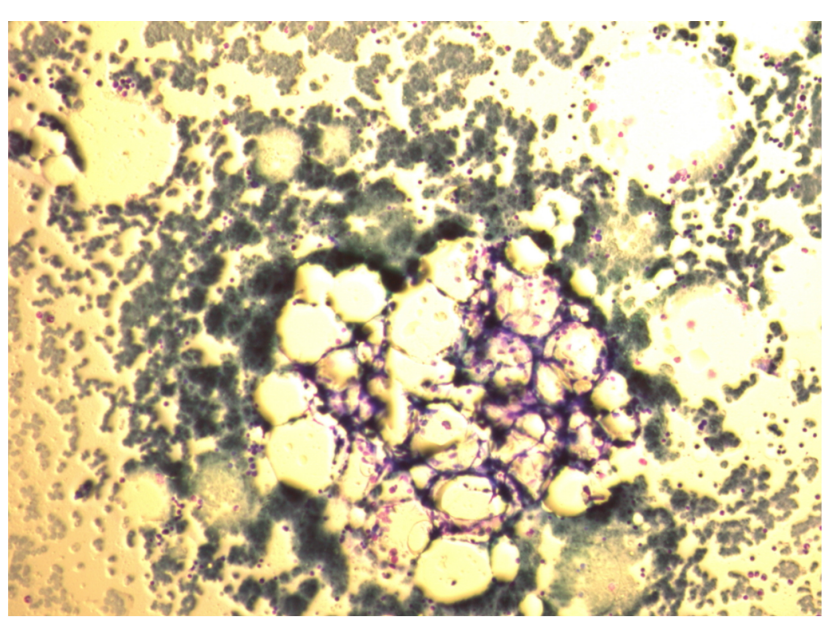

Figure 2. Bone marrow sample (19-6-2013). Compatible with a medullary aplasia (May-Grünwald-Giemsa colouring; magnification $200 \times$ ). 
Considering the importance of bone marrow failure, the therapeutic choice was oriented towards an allogenic bone marrow grafting. Thanks to a perfect HLA-compatibility between the patient and her brother, she could undergo, after some support transfusions, a selected CD34 allograft on November 22th 2013 in the Unity of Medullary Transplantation of the CHU-Sart-Tilman (University of Liège; Belgium; Professor Yves Beguin). Tolerance to the procedure was simple, just shadowed with an episode of fever with cutaneous rash and 2 episodes of Clostridium difficile colitis. An acute renal failure attributed to toxicity of tacrolimus was also quickly resolutive.

During follow-up, a poor medullary and lymphocytic chimerism but a clear improvement of pancytopenia and a progressive disappearance of GPI-deficient clone (undetectable from november 2014) were ascertained. Cyanocobalamin deficiency definitively attributed to previous gastroplasty had to be corrected with monthly injections. Despite a limited therapeutic compliance to post-grafting medications (tacrolimus, aciclovir, co-trimoxazole) a progressive weaning from march 2015 and even a complete withdrawal of tacrolimus from may 26th 2015 were possible. Hematological assessment remained normal from September 29th 2015 until now (October 10th 2017).

\section{Discussion}

On the contrary to other intrinsic abnormalities of erythrocyte, $\mathrm{PNH}$ is a rare acquired mutation of hematopoietic stem cell. This mutation is located on $\mathrm{X}$ chromosome at the level of phosphatidyl-inositol glycan (GPI) class A gene. ${ }^{[2-6]}$ This gene is required for GPI synthesis, a glycolipid derivative necessary to anchor number of proteins to cell surface. ${ }^{[3-5]}$ Thus descending cells from affected stem cells (erythrocytes, granulocytes, platelets, monocytes and lymphocytes) will be deficient for all GPI-anchored proteins. ${ }^{[2-6]}$ Among twenty deficient proteins, DAF (decay accelerating factor) or CD 55 and MIRL (membrane inhibitor of reactive lysis) or CD 59, both primary regulators of complement at the level of erythrocyte membrane will be of peculiar interest. ${ }^{[2-6]}$ In fact their lowering will refer to intravascular complement mediated hemolysis, one of the 3 key symptoms of the disease. ${ }^{[2-6]}$ Effectively the frequency of hemolytic crises is in direct relation to PGI clone size and LDH serum levels. Ecuzulimab (Eculizumab ; Solirix R, Alexion Pharmaceuticals), ${ }^{[6-9]}$ a humanized monoclonal antibody recently developed is able to block specifically terminal activation of the complement cascade at the C5 level. Prevention of C5 clivage will block C5a induction, a pro-inflammatory molecule, and C5b, initial substrate for erythrocytes cell membrane attacking complexes. Despite individual variations, ecuzulimab will improve pa-

Published by Sciedu Press tients quality of life by reducing almost completely intravascular hemolysis. ${ }^{[7-9]}$

Actual diagnosis of PNH, relies on flow cytometry determination of CD55 and CD59 markers at the erythrocyte membrane. Another interesting marker is the FLAER (fluorescentlabeled proaerolysin). Aerolysin, the principal virulence factor of aeromonas hydrophilic bacteria, is secreted as an inert protein, proto-aerolysin, which present a specific affinity for $G P I$-anchor; thus absence of its expression is pathognomonic of the presence of a GPI-deficient clone. ${ }^{[2-6]}$ For our patient, these 3 markers were confirmed positive at the blood and bone marrow levels.

The second usual symptom of PNH is characterized thrombophilia. ${ }^{[1-6]}$ Various mechanisms have been evoked to explain its physio-pathology. It seems that continuous activation of complement at the platelets surface will produce release of thrombinogen particules enriched in phosphatidylserine which can fix factor $\mathrm{V}$ and prothombinase complexes. ${ }^{[5,6]}$ On another hand, intravascular hemolysis will exert a direct toxicity on endothelial cells membrane. ${ }^{[3,5,10]}$ These will release Willebrand and VIII factors and express inhibitors of tissular factor and plasminogen activator. ${ }^{[6-9]}$

A third component of the PNH syndrome tripod is medullary insufficiency, affecting $48 \%$ of patients. ${ }^{[3,5,10]}$ Its physiopathology seems to be complex, 2 phenomenons being necessary to obtain bone marrow aplasia. ${ }^{[3,5,10]}$ In fact, PIG-A mutation will select PNH stem cells but it will be unable per se to favour their expansion; this last figure will be mediated through micro-environment auto or- dysimmunity. ${ }^{[3,5,10,11]}$ In other respects, oligoclonal $\mathrm{T}$ proliferation and apoptosis resistance of lymphocytes in proportion to PIG-A mutated stem cells levels have been evidenced. ${ }^{[5,10]}$

For our patient, thrombotic accidents were absent. Hemolysis was moderate (normal LDH levels), thus ecuzulimab was not retained, as targeted monoclonal antibody. ${ }^{[6-10]}$ In fact clinical presentation was dominated by a progressive medullary failure requiring some transfusional supports before allograft.

The French Hematological Society has reported a significant improvement of PNH survival along time. ${ }^{[3]}$ If in 1996 the median survival was 14.6 years, in 2008 it attained 22 years. ${ }^{[2]}$ The factors of reduced survival were: diagnosis before 1996; age over 40; no treatment during first year; progression towards a bi- or pancytopenia; supervening of thrombotic complications and rare (4\%) evolution to myelodysplasic syndrome or acute leukemia ${ }^{[2]}$. Allogenic bone marrow grafting is the only curative treatment of $\mathrm{PNH}$ with a success varying from $50 \%$ to $70 \% .^{[12]}$ A non myeloablative 
regimen is preferable in young people wishing to preserve their fertility. ${ }^{[12]}$ In the case of our patient, a complete sustained remission of PNH disease has been obtained with the administered therapy.

\section{Conclusion}

We had the privilege to diagnose a Marchiafava-Micheli syndrome or PNH in a young woman. The diagnosis was obtained after search for specific markers of the disease as the clinical presentation was initially dominated by vitamin B12 deficiency. Medullary deficiency being ultimately the principal symptom, a selected CD34 fully compatible allograft allowed obtention of a complete remission of affection with progressive disappearance of PNH clone and thus potential clinical cure.

\section{CONFlicts OF InTEREST Disclosure}

The authors declare no conflicts of interest.

\section{REFERENCES}

[1] Parker CJ. Paroxysmal nocturnal hemoglobinuria: an historical overview. Hematology Am Soc Hematol Educ Program. 2008; 1: 93 103. https://doi.org/10.1182/asheducation.2008.1.93

[2] Parker CJ. Update on the diagnosis and management of paroxysmal nocturnal hemoglobinuria. Hematology Am Soc Hematol Educ Program. 2016; 1: 208-216. https://doi .org/10.1182/asheduca tion-2016.1.208

[3] Peffaut de Latour R, Mary JY, Salanoubat C, et al. Paroxysmal nocturnal hemoglobinuria natural history of disease subcategories. Blood. 2008; 112(8): 3099-106. https://doi.org/10.1182/blood-2 008-01-133918

[4] Verreddy P. Hemoglobinuria misidentified as hematuria: review of discolored urine and paroxysmal nocturnal hemoglobinuria. Clinical medical insights: Blood Disorders. 2013; 6: 7-17. https: //doi.org/10.4137/CMBD.S11517

[5] Devalet B, Mullier F, Chatelain B, et al. Pathophysiology, diagnosis and treatment of paroxysmal nocturnal hemoglubinuria: a review. Europ J Haematol. 2015; 95(3): 190-8. https://doi .org/10.1 111/ejh12543

[6] Luzzatto L. Recent advances in the pathogenesis and treatment of paroxysmal nocturnal hemoglobinuria. F1000Research. 2016; 5(F1000 Faculty Rev): 209. https://doi.org/10.12688/110 00research.7288.1
[7] Hillmen P, Young NS, Schubert J, et al. The complement inhibitor eculizumab in paroxysmal nocturnal hemoglobinuria. N Engl J Med 2006; 355(12): 1233-43. PMid:16990386. https ://doi .org/10 $.1056 /$ NEJMoa061648

[8] Brodsky RA, Young NS, Antonioli E, et al. Multicenter phase 3 study of the complement inhibitor eculizumab for the treatment of patients with paroxysmal nocturnal hemoglobinuria. Blood. 2008; 111: 18407. PMid:18055865. https://doi .org/10.1182/blood-2007-0 6-094136

[9] Al-Ani F, Chin-Yee I, Lazo-Langner A. Ecuzulimab in the management of paroxysmal nocturnal hemoglobinuria: patient selection and special considerations. Ther Clin Risk Manag. 2016; 12: 1161-70. PMid:27536121. https://doi.org/10.2147/TCRM.S96720

[10] Griffin M, Munir T. Management of thrombosis in paroxysmal nocturnal hemaglobinuria: a clinician's guide. Ther Adv Hematol 2017; 8(3): 119-126. https://doi.org/10.1177/2040620716 681748

[11] Ishiyama K. Immune pathophysiology of acquired aplastic anemia. Rinsho Ketsueki. 2016; 57(5): 525-30. https://doi.org/10.114 06/rinketsu. 57.525

[12] Brodsky RA. Stem cell transplantation for paroxysmal nocturnal hemoglobinuria. Haematologica. 2010; 95(6): 855-6. https : //doi . org/10.3324/haematol.2010.023176 